\title{
ИССЛЕДОВАНИЕ СРЕДСТВ ОТСЛЕЖИВАНИЯ АКТИВНОСТИ ПОЛЬЗОВАТЕЛЕЙ В СИСТЕМАХ ЭЛЕКТРОННОГО ОБУЧЕНИЯ
}

\section{THE RESEARCH OF USER ACTIVITY TRACKING TOOLS IN E-LEARNING SYSTEMS}

N. Matrosova

Summary. Tracking user activity can help you design recommendation engines. Therefore, in this article, the author decided to investigate the tools that recognize and record user actions in e-learning systems (for example, LMS Moodle). In particular, the article considers the tools that allow obtaining information about the general activity of the user and about the movement of the mouse pointer. It also touches on the issue of the correlation between gaze movement and the mouse pointer. The article focuses on simple monitoring tools that can be used by any owner of an Internet service.

Keywords: e-learning system, mouse tracking, action tracking system, activity monitoring, eye tracking.

\author{
Матросова Наталия Дмитриевна \\ Аспирант, ФГАОУ ВО «Национальный \\ исследовательский университет ИТМО» \\ ndmatrosova@itmo.ru
}

Аннотация. Отслеживание активности пользователей может помочь при разработке рекомендательных механизмов. Поэтому в своей статье автор решил исследовать средства, которые распознают и фиксируют действия пользователей в системах электронного обучения (на примере LMS Moodle). В частности, рассмотрены средства, позволяющие получать информацию об общей активности пользователя и о движении указателя мыши. Также затронут вопрос о корреляции движения взгляда и указателя мыши. Акцент в статье сделан на простые средства мониторинга, которыми может воспользоваться любой владелец интернет-сервиса.

Ключевые слова: система электронного обучения, трекинг мыши, система отслеживания действий, мониторинг активности, айтрекинг.

на определенном дистанционном курсе, выполнении заданий, тестов и/или создании собственного контента.

Под отслеживанием активности пользователей понимается процесс отслеживания действий и поведение пользователей. Очень близкое к данному понятию "система контроля действий пользователя" имеет более жесткую функциональность - помимо отслеживания действий, такая система может еще и предотвращать нежелательную активность. Автор статьи остановится именно на отслеживании активности пользователей, или "мониторингом активности пользователей".

Одной из главных особенностей мониторинга активности является юридическое регулирование: любая фиксация действий должна происходить с предварительным уведомлением пользователей и получением от них согласия. В случае, если пользователь отказывается предоставить такое согласие, необходимо предложить ему покинуть интернет-страницу или не записывать его действия.

После решения юридических вопросов, имеет смысл решить каким именно образом будет отслеживаться информация о поведении. На данный момент, можно разделить способы отслеживания действия пользователя на две группы: 


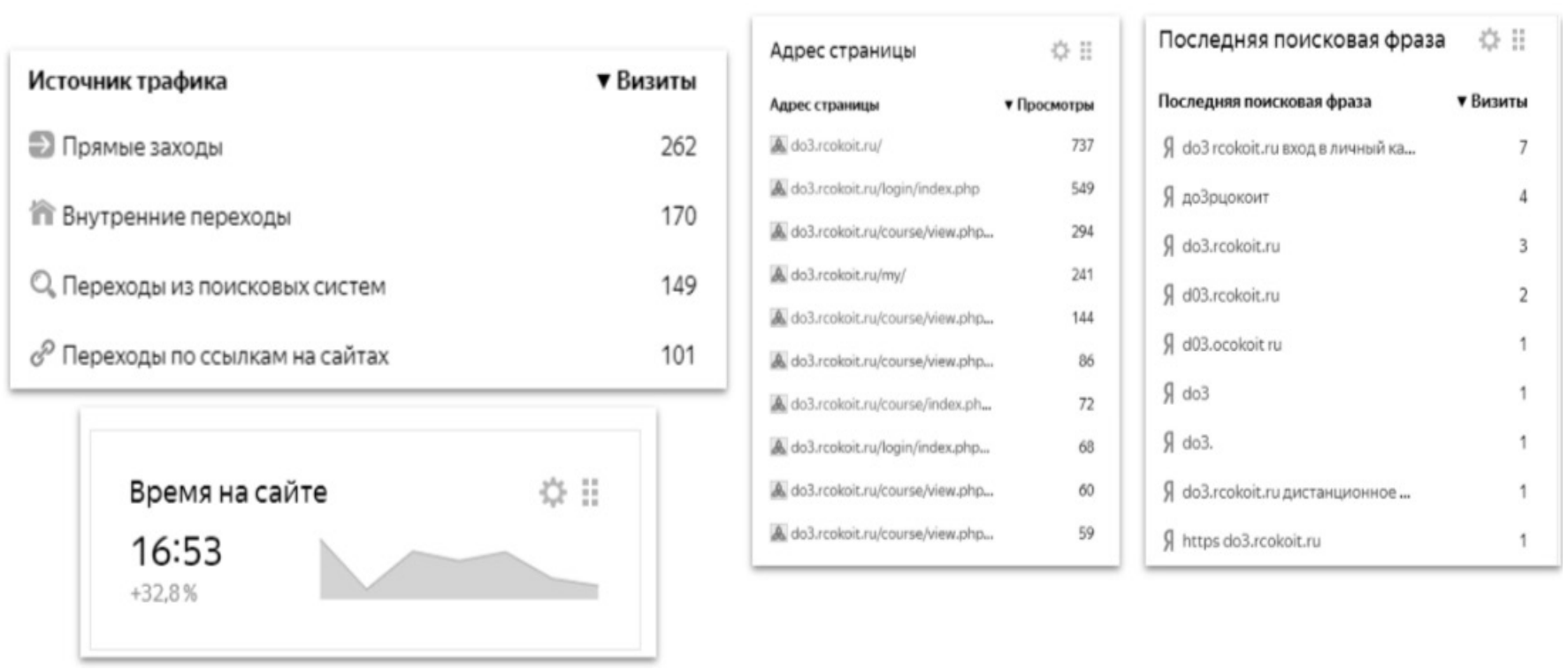

Рис. 1. Яндекс.Метрика - общая информация об активности пользователей

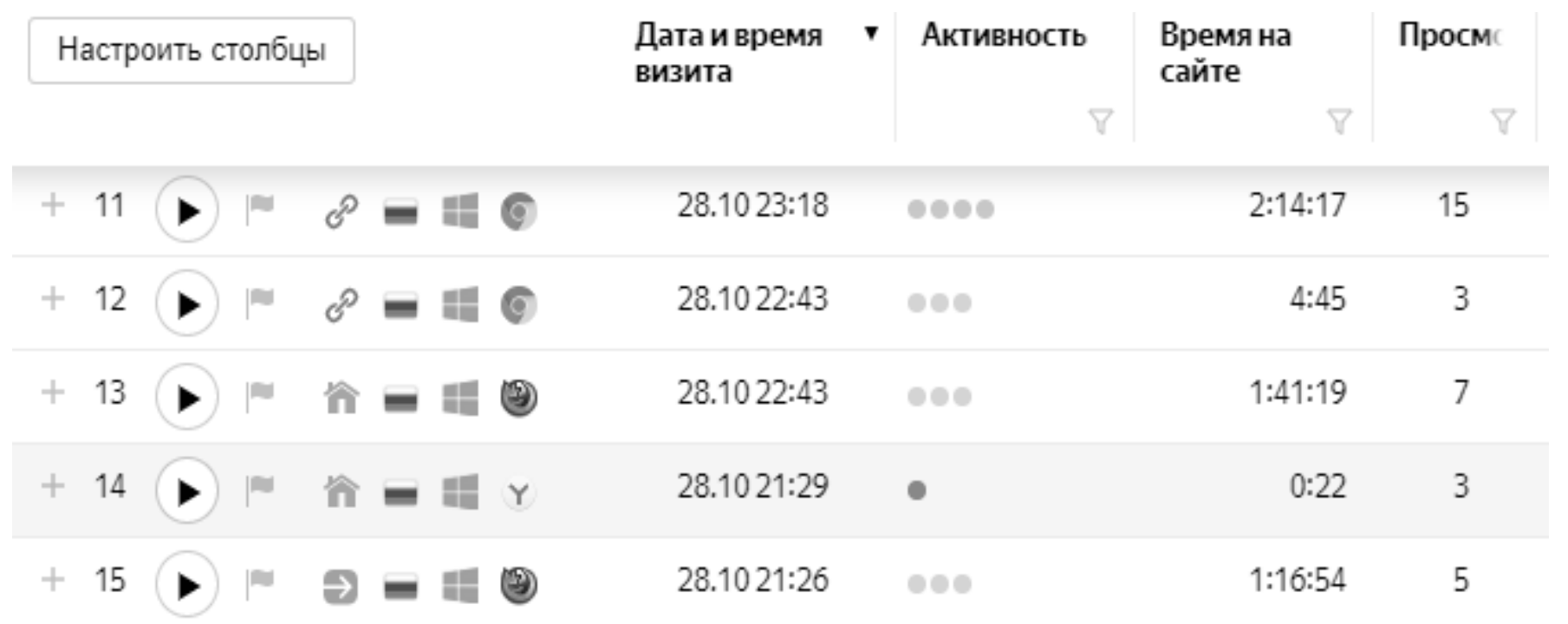

Рис. 2. Вебвизор Яндекс. Метрика - пример полученных записей сессий

- с помощью специальных устройств;

- с использованием специального программного обеспечения и онлайн-сервисов.

К специальным устройствам могут относиться такие устройства, как система отслеживания движений головы, системы захваты движений, отслеживатель глаз (айтрекеры) и т.п. Остановимся немного подробнее на айтрекинг - процесс, который изучает зрительное восприятие. Данная технология зародилась еще в XIX в. Айтрекеры представляют собой устройство, которое распознает и записывает точки фиксации и пути пере- мещения взгляда. [3] Недостатком использования таких устройств является их обязательное наличие на компьютере, с которого работает пользователь. Таким образом, в дальнейшем тексте автор остановится на возможностях специального программного обеспечения (ПО) и онлайн-сервисов для отслеживания активности пользователя.

Как уже упоминалось ранее, с помощью специальных средств можно фиксировать движения головы, зрачков, конечностей тела, но с использованием только ПО список действий для отслеживания сокращает- 
ся: чаще всего в таких ситуациях отслеживаются активность пользователя на сайте и/или трекинг указателя мыши.

Рассмотрим подробнее, во-первых, активность пользователя на сайте - данные, которые могут в себе содержать данные о:

- точке входа пользователя, то есть откуда он перешел на данную страницу: с другой страницы сайта, с поисковой системы, с социальной сети и т.д.;

- точка выходе, то есть странице, которая была последней в сессии пользователя;

- переходы между страницами - сколько переходов он сделал между страницами, за какое время и т.п.;

- времени входа на сайт (адат и время);

- сведения об браузере и устройстве пользователя

- другое в зависимости от средств мониторинга.

Самыми известными средствами мониторинга активности пользователей на сайтах являются Яндекс. Метрика и Google.Аналитика, которые с помощью специально-подключаемого счетчика получают доступ к информации о пользователях, заходящих на сайт. Оба данных средства мониторинга имеют схожие функциональные возможности: наборы базовых отчётностей, фильтрацию данных, возможности визуализации. [4]

На рисунке 1 приведен пример общей информации об источнике трафике и количестве просмотров определенных страниц с использованием Яндекс.Метрики. По полученным общим данным можно сделать вывод о наиболее частых опечатках при переходе на портал через поисковую систему «яндекс», среднем количестве времени на сайте и страницах, которые чаще всего посещают.

Использование перечисленных средств может помочь определить причину отмены выполнения какого-либо действия на сайте, определить наиболее и наименее популярные маршруты пользователей, а также выявить их соответствие задуманным.

Также стоит заметить, что многие системы электронного обучения (СЭО) имеют собственные средства мониторинга активности пользователей. Например, LMS Moodle обладает внутренним журналом действий и позволяет получать доступ к расширенной статистике [6], а платформа EdX - модуль отслеживания активностей пользователей. [5] Особенностью таких внутренних средств мониторинга является невозможность получения данных о точках входа пользователя или количестве переходов с социальных сетей.
Во-вторых, исследуем средства отслеживания указателя мыши (трекинг мыши, трекинг указателя мыши). Отслеживание указателя мыши могут включать в себе информацию о:

- непосредственно движении указателя мыши с фиксированием координат относительно экрана устройства пользователя;

- простое - время неактивности указателя;

- кликах - количество и место кликов, а также информация о клике левой или правой кнопки мыши;

- скроллинге - информация о пролистывании экрана с использованием колеса «прокрутки»;

- также может о скорости перемещении.

Движения указателя мыши хорошо фиксируется с помощью вебвизора Яндекс.Метрики - происходит запись каждой сессии каждого пользователя, которую можно потом просмотреть (См. Рисунок 2). Хотелось бы здесь акцентировать внимание, что, несмотря на то, что Вебвизор содержит в себе информацию для построения тепловых карт, карт кликов, это возможно не для всех сайтов. Портал, который используется как пример в данном исследовании, не обладает необходимыми условиями для построения автоматических карт.

Также особенностью Вебвизора является не хранение координат передвижения мыши, а воспроизведение движений пользователя в видео-формате.

Для LMS Moodle существует ряд расширений, которые могут быть установлены в систему, для фиксации координат указателя мыши. Отдельно хотелось бы остановиться на разработке [2]. Координаты движения мыши записываются в отдельную базу данных на сервере, что усложняют работу с данными.

Существуют исследования, которые анализируют соответствие движения взгляда и указателя мыши. В своем исследовании авторы [1] приходят к выводу, что точность сопоставления указателя и взгляда достаточно высока. Поэтому в исследованиях, в которых нет возможности использовать специальные устройства для трекинга взгляда, можно использовать ПО или сервисы для трекинга мыши.

В качестве вывода, автор хотел бы заметить, что в данной статье были рассмотрены самые распространенные средства отслеживания активности пользователей. Существует ряд решений, которые доступны на JavaScript, HTML и CSS - такие решения позволяют получать более точные, с точки зрения исследователя, данные, но при этом они сложнее из-за необходимости получать доступ к базам данных и требуют больше трудоемкости. 
ЛИТЕРАТУРА

1. Boi,Paolo \& Fenu, Gianni \& Spano, Lucio \& Vargiu,Valentino. (2016). Reconstructing User's Attention on the Web through Mouse Movements and Perception-Based Content Identification. ACM Transactions on Applied Perception. 13. 1-21. 10.1145/2912124. (URL: https://www.researchgate.net/publication/303711174_ Reconstructing_User's_Attention_on_the_Web_through_Mouse_Movements_and_Perception-Based_Content_Identification)

2. Purnama, Fajar \& Sukhbaatar, Otgontsetseg \& Choimaa, Lodoiravsal \& Usagawa, Tsuyoshi. (2020). Implementation of real-time online mouse tracking on overseas quiz session: From server administrator point of view. Education and Information Technologies. 25. 10.1007/s10639-020-10141-3.

3. Абабкова Марианна Юрьевна, Розова Наталья Константиновна К вопросу о месте технологии айтрекинга в российской высшей школе // Известия ВГПу. 2020. № 3 (146). URL: https://cyberleninka.ru/article/n/k-voprosu-o-meste-tehnologii-aytrekinga-v-rossiyskoy-vysshey-shkole (дата обращения: 03.06.2021).

4. Иванченко 0.В., Барауля Е.В. Веб-аналитика в формировании исследовательской информационно-коммуникационной инфраструктуры маркетинга отношений // Вестник РГЭУ РИНХ. 2020. № 1 (69). URL: https://cyberleninka.ru/article/n/veb-analitika-v-formirovanii-issledovatelskoy-informatsionnokommunikatsionnoy-infrastruktury-marketinga-otnosheniy (дата обращения: 03.06.2021).

5. Матросова Н.Д., Штенников Д.Г. Методы построения индивидуальных образовательных траекторий с использованием платформы массовых онлайн курсов Edx // Проблемы и перспективы внедрения свободного программного обеспечения в образовательных организациях Санкт-Петербурга. Матлы XII конференции - 2019.- - С. 41-46

6. Матросова Н.Д., Штенников Д.Г. Расширенная статистика LMS Moodle // Проблемы и перспективы внедрения свободного программного обеспечения в образовательных организациях Санкт-Петербурга. Мат-лы XI конференции - 2018. - C. 16-21

( ) Матросова Наталия Дмитриевна ( ndmatrosova@itmo.ru).

Журнал «Современная наука: актуальные проблемы теории и практики»

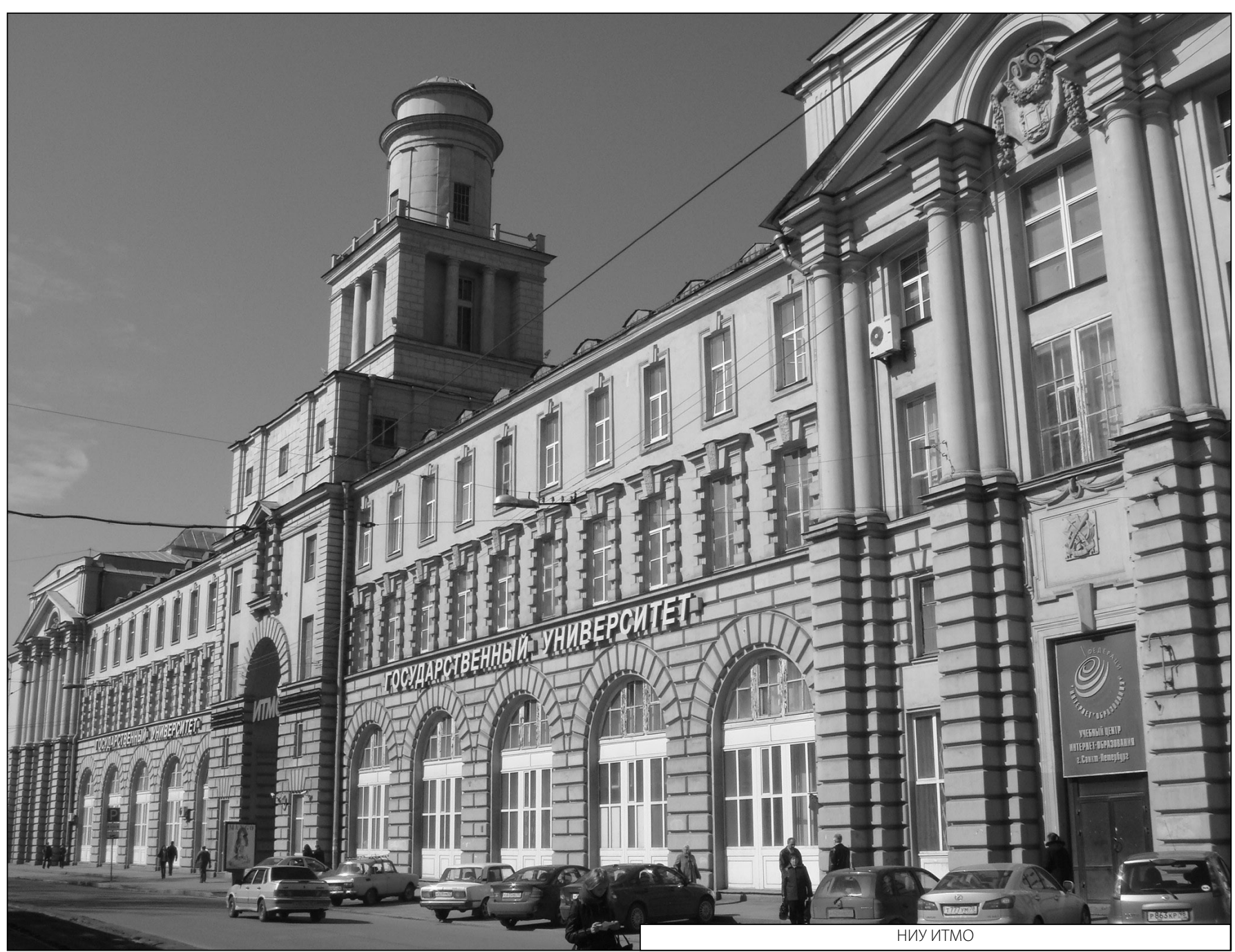

